\section{The leucine-rich repeat protein SUR-8 enhances MAP kinase activation and forms a complex with Ras and Raf}

\author{
Weiquan Li, ${ }^{1}$ Min Han, ${ }^{3}$ and Kun-Liang Guan ${ }^{1,2,4}$ \\ ${ }^{1}$ Department of Biological Chemistry, ${ }^{2}$ Institute \\ of Gerontology, University of Michigan Medical School, \\ Ann Arbor, Michigan 48109 USA; ${ }^{3}$ Department of Molecular, \\ Cellular, and Developmental Biology, Howard Hughes \\ Medical Institutes, University of Colorado, Boulder, Colorado \\ 80309 USA
}

Caenorhabditis elegans sur-8 encodes a positive regulator of Ras signaling. We investigated the mechanism by which the human Sur- 8 homolog can positively regulate Ras-MAP kinase signaling in mammalian cells. Sur-8 expression enhances Ras- or EGF-induced Raf and ERK activation but has no effect on ERK activation induced by active Raf or MEK. Furthermore, Sur-8 expression does not increase AKT or JNK activation. Sur-8 interacts with Ras and Raf and is able to form a ternary complex with the two proteins. Thus, Sur- 8 may function as a scaffold that enhances Ras-MAP kinase signal transduction by facilitating the interaction between Ras and Raf.

Received January 20, 2000; revised version accepted March 2, 2000.

Ras relays signals from receptor tyrosine kinases (RTKs) to activation of the mitogen-activated protein kinase (MAPK) pathway (van der Geer et al. 1994; Robinson and Cobb 1997). Ras directly interacts with and activates Raf, which phosphorylates and activates MEK. MEK is a dual-specific kinase that selectively phosphorylates and activates the extracellular signal-regulated kinase, ERK, a MAPK family member. Ras also interacts with numerous other downstream molecules, although the functional significance of many of the Ras-interacting proteins is not clear (Katz and McCormick 1997; Vojtek and Der 1998). However, it has been shown that PI3K and RalGDS are physiological targets of Ras (Rodriguez-Viciana et al. 1994; Urano et al. 1996).

Genetic studies in Caenorhabditis elegans and Drosophila demonstrate that the Ras-MAPK pathway is essential for signal transduction of RTK (Chang et al. 1994; Sternberg and Han 1998). Recent genetic studies have identified additional molecules that may regulate RasMAPK signaling. Sieburth et al. (1998) isolated sur-8 by screening for mutations that can suppress the Multivulva phenotype of an activated let-60 Ras mutation. Selfors et al. (1998) identified soc-2, which is identical to

[Key Words: Sur-8; RTK; Ras; Raf; ERK]

${ }^{4}$ Corresponding author.

E-MAIL kunliang@umich.edu; FAX (734) 763-4581. sur-8, by isolating suppressors of $c l r-1$, which encodes a receptor tyrosine phosphatase. soc-2 mutations also suppress the activity of an hyperactive mutation in egl-15 FGF receptor gene, suggesting that soc-2 functions in RTK signaling. Although single mutation of sur-8 in an otherwise wild-type background causes no obvious phenotype, mutation of sur-8 dramatically enhances the weak loss-of-function mutation in mpk-1 (Sieburth et al. 1998), indicating that sur-8 is required for optimal RasMAPK signaling. These properties of sur- 8 are similar to other worm genes such as ksr (Kornfeld et al. 1995; Sundaram and Han 1995) and sur-6 PP2A-B (Sieburth et al. 1999). Because double mutations in sur-8 and ksr essentially eliminate vulval induction in C. elegans, the functions of these two genes together are essential for Ras signaling.

The deduced amino acid sequence of sur- 8 contains multiple leucine-rich repeats (LRR), which are present in numerous proteins and may mediate protein-protein interaction (Kobe and Deisenhofer 1993). LRR is found in the yeast adenylyl cyclase (Kataoka et al. 1985), which is a direct downstream target of Ras, and the LRR sequences in the yeast adenylyl cyclase mediate its interaction with Ras (Field et al. 1990; Suzuki et al. 1990). Genetic data suggest that sur-8 functions in RTK signaling, most likely downstream of Ras and upstream of Raf. Both C. elegans and mammalian Sur-8 proteins were shown to directly bind to Ras (Sieburth et al. 1998); however, the biochemical mechanism of Sur-8 in Ras signaling has not been determined.

The human Sur-8 cDNA can functionally complement the sur-8 mutation in C. elegans (Sieburth et al. 1998), suggesting that the Sur-8 function is conserved. We tested the biochemical functions of human Sur- 8 on RasMAPK signaling. Data in this report suggest that Sur-8 may modulate the potency and specificity of Ras signaling.

Results

Sur-8 enhances the activation of ERK by EGF or Ras

We tested the effect of expressing human Sur-8 on the activity of ERK in mammalian cells. Myc-tagged ERK1 was cotransfected into 293 cells with Ras and Sur-8 followed by immunoprecipitation and an in vitro kinase assay. Sur-8 alone had no significant effect on ERK activity (Fig. 1A). However, in the presence of Ras, Sur-8 significantly enhanced the ability of Ras to activate ERK. The enhancement of ERK activity by Sur- 8 was most significant at low concentrations of Ras signal (Fig. 1A). Sur-8 had similar effects on ERK activation in response to H-Ras, K-Ras, and N-Ras (Fig. 1B). Sur-8 enhanced ERK activation in a dose-dependent manner (Fig. 1B).

The effect of Sur-8 on EGF-stimulated ERK activation was also examined. At low EGF concentrations, cotransfection of Sur-8 significantly increased EGF-induced ERK activity (Fig. 1C). However, at a high concentration of EGF (100 ng/ml), maximum ERK activation was ob- 


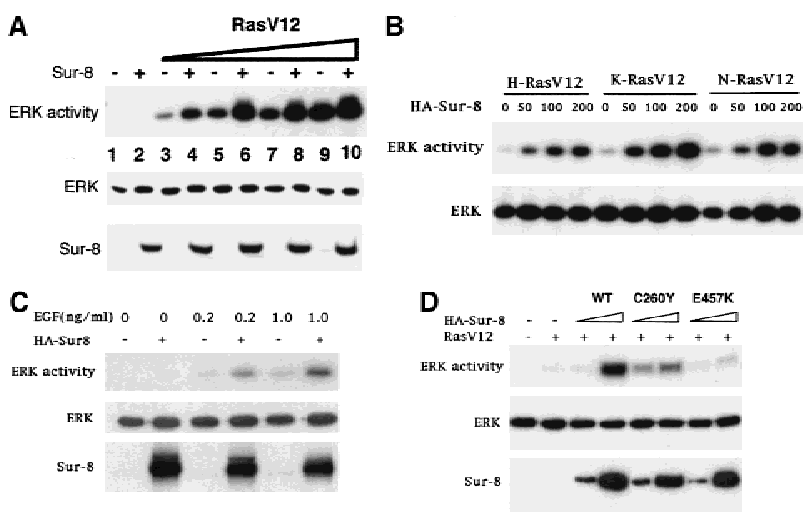

Figure 1. Sur-8 enhances ERK activation. (A) Myc-tagged ERK1 (200 ng) was cotransfected with increasing amounts of RasV12 in the presence or absence of Sur-8 (500 ng) as indicated. The amounts of RasV12 (all Ras plasmids used are K-Ras except as described) used for transfection are 0 (lanes 1,2), $5 \mathrm{ng}$ (lanes 3,4), $10 \mathrm{ng}$ (lanes 5,6), $20 \mathrm{ng}$ (lanes 7,8), and 50 ng (lanes 9,10). Myc-ERK was immunoprecipitated and kinase activity was determined using GST-Elk-1 as a substrate (top). Myc-ERK in the immunoprecipitate was determined by anti-ERK Western blot (middle). Expression of HA-Sur-8 in cell lysates was detected by anti-HA Western blot (bottom). (B) Dose-dependent effect of Sur-8 on ERK activity. Cells were cotransfected with $1 \mathrm{ng}$ of H-RasV12, K-RasV12, or N-RasV12, and 200 ng of Myc-ERK. The amount of HA-Sur-8 (ng DNA) cotransfected is indicated. (C) Sur-8 enhances EGF-stimulated ERK activity. The 293 cells were cotransfected with 200 ng of Myc-ERK and 500 ng of HASur-8. After cells were starved in serum-free medium overnight, the cells were stimulated for $5 \mathrm{~min}$ by indicated concentrations of EGF. Myc-ERK was immunoprecipitated and activity was determined. (D) Mutations at Cys-260 and Glu457 of Sur-8 compromised its functions in ERK activation. Sur- 8 or mutants $(50$ and $500 \mathrm{ng}$ ) were cotransfected with Myc-ERK and RasV12. Immunoprecipitated ERK activity and expression levels are shown at top and middle, respectively. HA-Sur-8 expression was detected as above (bottom).

served and Sur-8 expression had no significant effect (data not shown). Together, these results indicate that Sur-8 has a positive function in RTK and Ras-induced ERK activation.

A genetic screen isolated two loss-of-function mutants of C. elegans sur-8 as Ras suppressors (Sieburth et al. 1998). The corresponding mutations, C260Y and E457K, were created in human Sur-8, and their abilities to enhance ERK activity were examined. Both C260Y and E457K mutants significantly reduced Sur-8's ability to enhance Ras-stimulated ERK activation (Fig. 1D). Thus, the ability of Sur-8 to enhance the ERK activation may be relevant to its in vivo function as a positive modulator of the Ras-MAPK signaling pathway.

\section{Sur-8 acts to up-regulate Raf activity}

To determine at which step Sur-8 affects ERK activation in mammalian cells, we examined the effect of Sur-8 on ERK activation induced by active Raf or active MEK. Sur-8 had no significant effect on ERK activation stimulated by a constitutively active MEK (Fig. 2A; Mansour et al. 1994) , suggesting that Sur-8 functions upstream of MEK. Sur-8 showed no significant effect on either V-Raf (Howe et al. 1992; Kyriakis et al. 1992) or active B-Rafinduced (A. Vojtek, unpubl.) ERK activation (Fig. 2B), supporting the notion that Sur-8 acts upstream of Raf. To test this hypothesis further, we examined whether Sur-8 enhances Raf activation. Sur-8 enhanced RasV12-stimulated, as well as EGF-induced, Raf activity (Fig. 2C). Therefore, these data are consistent with a model that Sur-8 functions upstream of Raf.

Sur-8 does not enhance activation of AKT or other MAPK by RasV12

Mammalian cells contain several MAPK pathways. To test the effect of Sur-8 on other MAPK, we determined JNK activity when Sur-8 was coexpressed. JNK is strongly activated by environmental stress such as anisomycin but is weakly activated by Ras (Fig. 2D; Derijard et al. 1994; Kyriakis et al. 1994). JNK activation induced by RasV12 was not enhanced by Sur-8 cotransfection (Fig. 2D). Similarly, the activation of JNK by anisomycin was not augmented by Sur-8 cotransfection. Moreover, Sur-8 had no effect on p38 MAPK activation (data not shown).

In addition to the Raf-ERK pathway, Ras activates multiple downstream targets (Vojtek and Der 1998). For example, Ras activates PI3K, thereby leading to activation of the protein kinase AKT (Marte and Downward 1997). Coexpression of Sur-8 had no significant effect on Ras-induced AKT activity (Fig. 2E). These results further demonstrate that Sur-8 specifically enhances the RasERK pathway.

\section{Sur-8 interacts with Ras and Raf}

Previous data have indicated that Sur-8 has strong interaction with K-Ras and N-Ras and weak interaction with H-Ras (Sieburth et al. 1998). We further tested the interaction between Sur-8 and Ras or other GTPases (Table 1). Sur-8 interacts with all three Ras isoforms by the yeast two-hybrid assays (Table 1) and coimmunoprecipitations (data not shown). In addition, Sur-8 interacts with the activated RasV12 mutants but not the dominant-negative RasN17 mutants. Ras interacts with downstream targets via its effector domain. We tested the interaction between Sur-8 and several Ras effector domain mutants. Mutations of Y32F, P34G, T35S, V45E, and A59E in RasV12 eliminated its interaction with Sur-8 (Table 1, an example is shown in Fig. 3A) without affecting Raf binding. Mutation of E37G in Ras abolished binding to both Sur-8 and Raf. Many Ras targets, including Raf and RalGDS, are also able to interact with Rap (Macara et al. 1996; Vojtek and Der 1998), a closely related member of the Ras family GTPases. Sur-8 showed no interaction with Rap,TC21, which interacted with Raf in the same assay, or Rac1. Our results demonstrate that Sur-8 specifically interacts with Ras but not with other related small GTPases and mutations of the Ras effector domain abolish its interaction with Sur-8. Furthermore, only the 


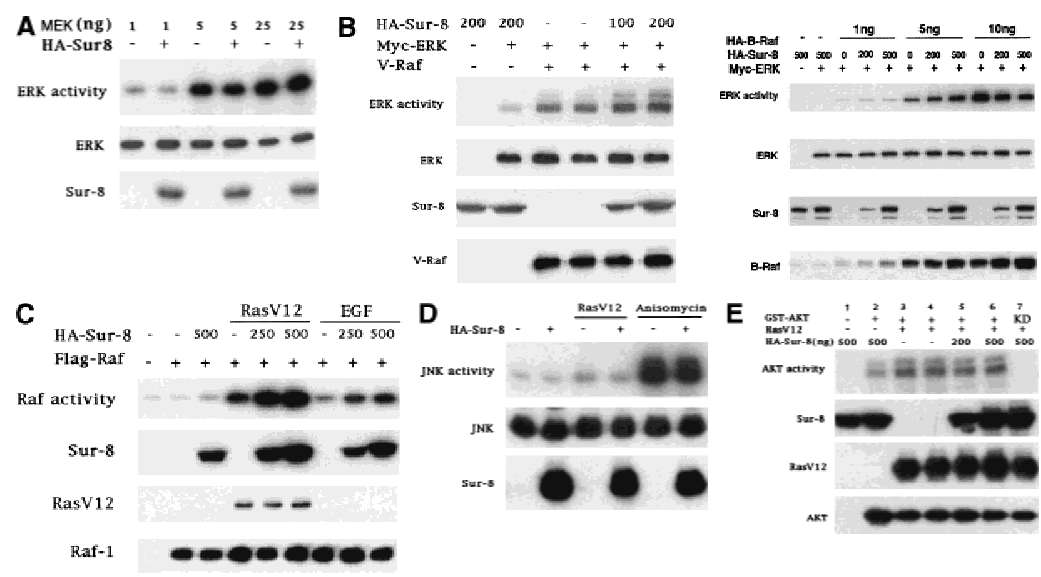

Figure 2. Sur- 8 specifically enhances the ERK pathway and functions upstream of Raf. (A) Sur-8 has no effect on MEK-induced ERK activation. The 293 cells were cotransfected with $500 \mathrm{ng}$ of HA-Sur-8, $200 \mathrm{ng}$ of Myc-ERK and varied amounts of a constitutively active MEK mutant as indicated. ERK activity was determined as in Fig. 1A. (B) Sur-8 has no effect on Raf-induced ERK activation. Myc-ERK (200 ng) was cotransfected with V-Raf (10 ng, left) or varied amounts of active B-Raf mutant (right). Cotransfection of Sur-8 did not further increase ERK activity. $(C)$ Sur-8 enhances Raf activity in response to RasV12 or EGF stimulation. 293 cells were cotransfected with 400 ng of Flag-Raf, 20 ng of HA-RasV12, and varied amounts of HA-Sur-8 as indicated. Cells were stimulated with EGF for $3 \mathrm{~min}$ where indicated. Flag-Raf was immunoprecipitated with anti-Flag and activity was determined by the coupled kinase assay. Expression of Sur-8 and Ras in lysates and the levels of Raf in the immunoprecipitates were confirmed by Western blot. $(D)$ Sur-8 has no effect on JNK activation. HA-JNK (300 ng) was transfected into cells. Cotransfection with RasV12 or treatment with anisomycin was used to stimulate JNK activity. HA-JNK was immunoprecipitated and assayed for kinase activity using GST-Jun as a substrate. (E) RasV12-induced AKT activation is not enhanced by Sur-8. Wild-type GST-AKT (100 ng) was cotransfected into cells with HA-RasV12 and varied amounts of Sur-8 as indicated. GST-AKT were purified and kinase activity was determined using histone $2 \mathrm{~B}$ as a substrate. (Lane 7) Negative control of the kinase dead mutant (KD) of GST-AKT.

active mutant RasV12 but not the dominant-negative mutant RasN17 shows a positive interaction with Sur-8.

The in vivo interaction between Sur-8 and Ras was tested by coimmunoprecipitation experiments. When cotransfected into 293 cells, HA-Sur-8 and Ras were reciprocally coimmunoprecipitated (Fig. 3B).

Our biochemical data indicate that Sur-8 might function between Ras and Raf, although no positive interaction between Raf and Sur- 8 was observed by the yeast two-hybrid assay (data not shown). We tested the possibility that Sur-8 may interact with Raf and other molecules acting downstream of Ras, and found that HASur-8 was coimmunoprecipitated with Raf (Fig. 3C). Reciprocal immunoprecipitation confirmed the interaction between Sur- 8 and Raf. Moreover, endogenous Raf and Sur-8 proteins interact in vivo. Anti-Sur-8 antibody coimmunoprecipitated endogenous Raf (Fig. 3E). As a control, anti-Ras antibody also coimmunoprecipitated Raf, whereas the control antibody did not. Raf consists of an amino-terminal domain, which is responsible for interaction with Ras (Vojtek et al. 1993), and a carboxy-terminal kinase domain. The carboxy-terminal domain $(\Delta)$ but not the amino-terminal domain $(\Delta \mathrm{C})$ of Raf retained a positive interaction with Sur-8 (Fig. 3D) These results show that different Raf domains are responsible for interacting with Ras and Sur-8. However, our data cannot exclude the possibility that other proteins mediate the interaction between Sur- 8 and Raf.

\section{Sur-8 can form a complex with Ras and Raf}

The above results demonstrate that Ras, Raf, and Sur-8 can interact with each other. A possible mechanism by which Sur-8 stimulates Raf activation is by promoting Ras-Raf complex formation. To test this hypothesis, Ras-Raf interaction was examined in the presence or absence of Sur-8 coexpression. Sur-8 significantly increased the amount of RasV12 copurified with GST-Raf (Fig. 4A). However, neither Sur-8C260Y nor E457K mutant can enhance the interaction between Ras and GST-Raf (Fig. 4B). These results suggest that the ability of Sur- 8 to enhance RasRaf interaction correlates with its activity to enhance Ras signaling.

To further confirm the formation of RasRaf-Sur-8 complex, we performed in vitro binding experiments using purified GST-Ras and cell lysates containing Raf and Sur-8. Addition of Raf enhanced the association between GST-Ras and Sur-8 (Fig. 4C). The carboxy-terminal domain of Sur-8 is required for interaction with Ras but not required for Sur8-Raf interaction (Sieburth et al. 1998) (data not shown). A Sur- $8 \Delta \mathrm{C}$ mutant showed no interaction with Ras (Fig. 4D, lane 1). Addition of Raf enhanced the interaction between Sur- $8 \Delta \mathrm{C}$ and Ras, suggesting that Raf is capable of bridging this interaction (Fig. 4D).

Table 1. Interaction between Sur-8 and Ras mutants in the yeast two-hybrid system

\begin{tabular}{lcccc}
\hline Target bait & Sur-8 & Raf & PI3K & RalGDS \\
\hline K-RasV12 & ++++ & ++++ & ++++ & ++++ \\
K-RasN17 & - & - & - & - \\
K-RasV12Y32F & - & ++++ & - & - \\
K-RasV12P34G & - & ++++ & - & - \\
K-RasV12T35S & - & ++++ & - & - \\
K-RasV12E37G & - & - & ++++ & \\
K-RasV12V45E & - & ++++ & ++++ & \\
K-RasV12A59E & - & ++++ & - & \\
H-RasV12 & +++ & ++++ & ++++ & ++++ \\
H-RasN17 & - & - & - & - \\
N-RasV12 & ++ & ++++ & ++++ & ++++ \\
N-RasN17 & - & - & - & - \\
RapV12 & - & +++ & & +++ \\
RacV12 & - & - & & \\
TC21 & - & +++ & & \\
Sur-8 & - & - & - & \\
Lamin & - & - & & \\
\hline
\end{tabular}

(Blankspace) Experiments not performed. 
Li et al.

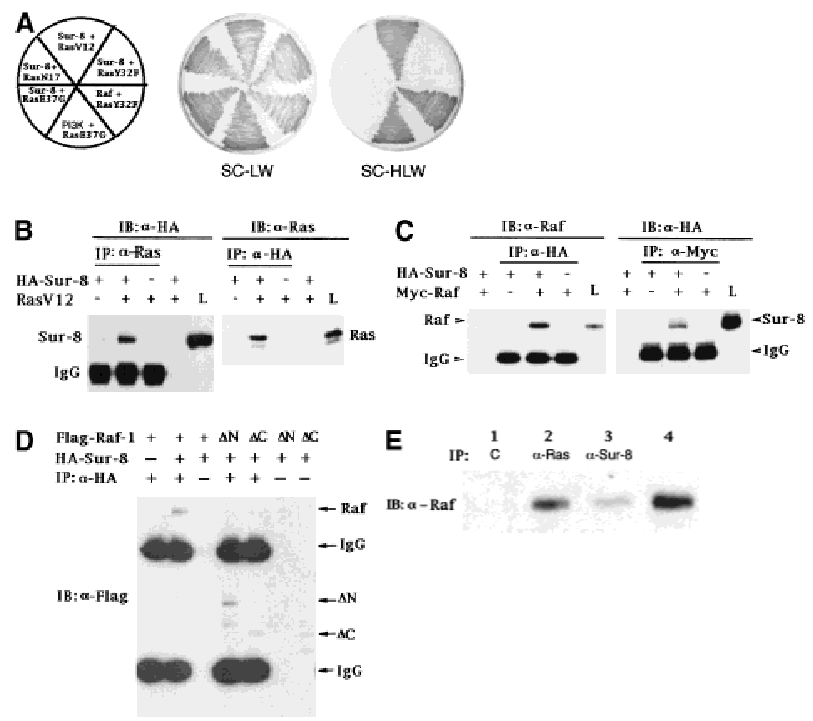

Figure 3. Sur-8 interacts with Ras and Raf. $(A)$ Interactions between Sur- 8 and Ras mutants in the yeast two-hybrid assays. Growth on SC-HLW medium indicates a positive interaction. RasY32F and RasE37G are in the RasV12 mutant background. (B) Coimmunoprecipitation between Sur-8 and RasV12. A total of $500 \mathrm{ng}$ of HA-Sur- 8 was cotransfected into 293 cells with 200 ng of RasV12 as indicated. Thirty-six hours after transfection, cells lysates were immunoprecipitated with anti-Ras antibody (left) or anti-HA (right) as indicated. The immunoprecipitates were blotted with anti-HA (left) or anti-Ras (right). (Lane L) Cell lysate controls. (IP, IB) Immunoprecipitation and immunoblot, respectively. (IgG) The immunoglobulin band. $(C)$ Interaction between Sur- 8 and Raf were determined by coimmunoprecipitation of transfected HA-Sur-8 and Myc-Raf. (Lane $L$ ) The cell lysate control. $(D)$ The carboxy-terminal domain of Raf interacts with Sur-8. Flag-tagged Raf full length, $\Delta \mathrm{N}$ (residues 325-648), $\Delta \mathrm{C}$ (residues 1-256) were cotransfected with HA-Sur-8. HASur- 8 was immunoprecipitated by anti-HA followed by Western blot with anti-Flag antibody. Negative controls without antiHA antibody were included. Bands corresponding to full length, $\Delta \mathrm{C}$, and $\Delta \mathrm{N}$ of Raf are indicated. (E) Coimmunoprecipitation between endogenous Sur-8 and Raf. The 293 cell lysates were immunoprecipitated with control serum (lane 1), anti-Ras (lane 2), or anti-Sur-8. The immunoprecipitates were blotted with anti-Raf. (Lane 4) Control of cell lysates.

We further characterized the formation of a Ras, Raf, and Sur-8 complex in vivo by sequential coimmunoprecipitation experiments. GST-Sur-8, HA-RasV12, and Myc-Raf were cotransfected into 293 cells. GST-Sur-8 and its associated proteins were purified, eluted with glutathione, and subjected to a second immunoprecipitation with anti-HA antibodies. The anti-HA immunoprecipitates were then subjected to Western blot with anti-Raf to detect Myc-Raf. Myc-Raf was present after sequential immunoprecipitation (Fig. 4E, lane 3). Experiments were also performed first by purification of GSTSur-8, followed by immunoprecipitation with anti-Myc to precipitate Myc-Raf, then blotted with anti-Ras to detect HA-Ras. Ras is present in the sample after sequential purification with GST-Sur-8 and Myc-Raf (Fig. 4E, lane 6). GST-Sur-8 may form a dimer and could complicate the interpretation of the above results. Similar
A

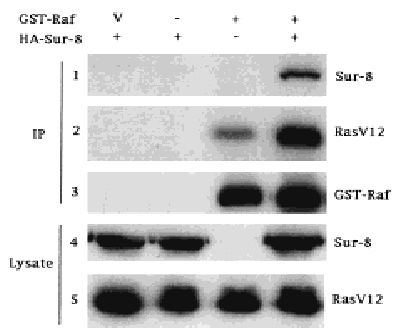

B

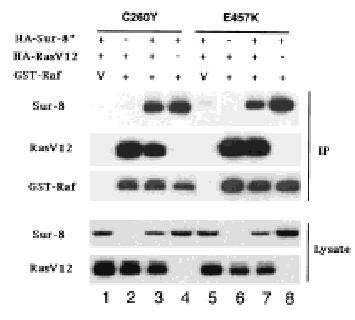

C
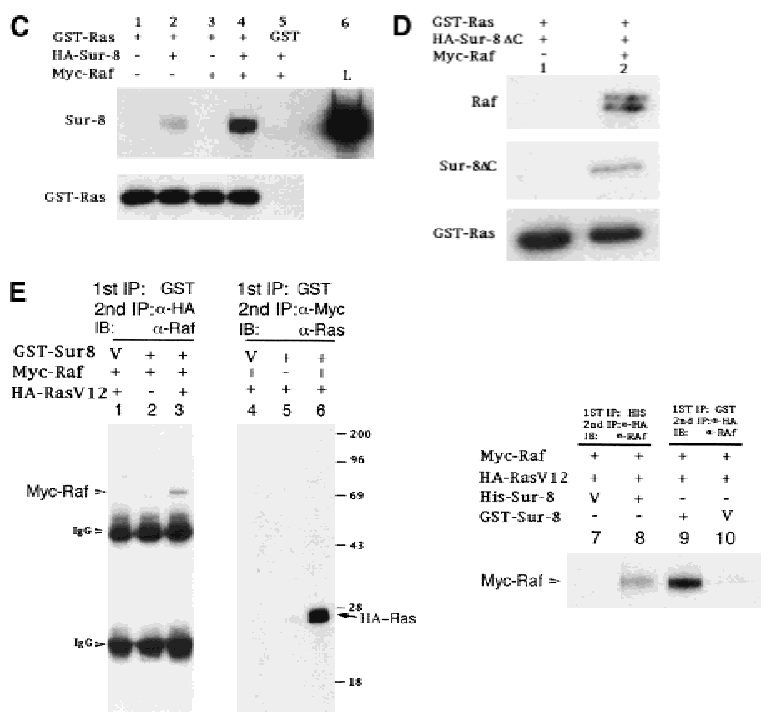

Figure 4. Sur-8 forms a complex with Ras and Raf. (A) Sur-8 enhances the interaction between Ras and Raf. HA-RasV12 was cotransfected with GST-Raf in the presence or absence of HASur-8. GST-Raf was purified by glutathione-agarose resin. The copurified Ras and HA-Sur-8 were detected by anti-HA for HARas (second panel from top) and HA-Sur-8 (top) Western blots. The GST-Raf in the precipitation was detected by an anti-Raf Western blot (middle). Expressions of HA-Sur-8 and HARasV12 in cell lysates were shown in bottom two panels. $(B)$ Sur-8C260Y and E457K mutants fail to enhance the interaction between Ras and Raf. Experiments are similar to A. (HA-Sur- $8^{*}$ ) Sur-8C260Y in lanes 1-4 and Sur-8E457K in lanes 5-8, respectively. $(C)$ Raf enhances Sur-8-Ras interaction in vitro. Cell lysates expressing HA-Sur-8 or Myc-Raf were mixed with purified GST-RasV12 protein $(5 \mu \mathrm{g})$. GST-RasV12 and associated proteins were purified by glutathione-agarose and Western blotted with anti-HA to detect HA-Sur-8 (top) and anti-GST to detect GST-RasV12 (bottom). (Lane 6) A control of cell lysate containing HA-Sur-8. (D) Raf bridges the interaction between Ras and Sur- $8 \Delta$ C. GST-Ras $(5 \mu \mathrm{g})$ was incubated with cell lysates containing HA-Sur- $8 \Delta \mathrm{C}$ (containing residues 1-366) in the absence (lane 1) or presence (lane 2) of Raf containing cell lysates. HA-Sur- $8 \Delta \mathrm{C}$ (middle) and Raf $(t o p)$ copurified by GSTRas were detected by Western blot. GST-Ras coprecipitated HA-Sur- $8 \Delta$ C only in the presence of Raf. $(E)$ Sur- 8 , Ras, and Raf form a ternary complex. The 293 cells were transfected with various plasmids as indicated. Cell lysates were subjected to purification by glutathione-agarose beads. The purified proteins were eluted by glutathione and subjected to immunoprecipitation with anti-HA for HA-Ras followed by Western blot with anti-Raf (left). Similarly, proteins eluted from glutathione-agarose beads were immunoprecipitated with anti-Myc for MycRaf followed by Western blot with anti-Ras (middle). (Right) Experimental results using His-Sur-8 (lane 8) along with GSTSur-8 (lane 9). His-Sur-8 complex was first purified by nickel agarose and eluted with imidazole. The eluted samples were immunoprecipitated with anti-Myc and followed by Western blot with anti-Raf. 
experiments using His-Sur-8 demonstrated that Raf protein was present after the sequential precipitations with nickel agarose (for His-Sur-8) and anti-HA (for HA-Ras) (Fig. 4E), consistent with experiments using GST-Sur-8. These data provide strong evidence that Ras, Raf, and Sur-8 together can form a ternary complex in vivo.

\section{Discussion}

Here we demonstrate that human Sur-8 can form a complex with Ras and Raf, and specifically enhances ERK activation. Our observations support the model that Sur- 8 acts between Ras and Raf. Sur- 8 alone does not significantly stimulate the ERK activity in the absence of EGF or RasV12. The effect of Sur-8 is most significant at low concentrations of EGF or Ras. Therefore, Sur- 8 enhances the signal strength of RTK and Ras by facilitating the Ras-Raf complex formation. Genetic properties of sur-8 in C. elegans are consistent with this notion. sur- 8 mutations alone do not cause obvious mutant phenotypes, but they are effective suppressors of activated Ras mutations and drastically enhance weak mutations in MAPK or Raf, indicating that Sur- 8 is required for optimal Ras-MAP kinase signaling (Sieburth et al. 1998). Our data demonstrate that Sur-8 may modulate the signal strength of Ras and RTK. Sur- 8 may also modulate the specificity of physiological responses. For example, Sur-8 selectively increases the signal from RTK or Ras to Raf but not to other downstream effectors such as PI3K.

The Ras-MAPK pathway represents one of the bestcharacterized signal transduction pathways. However, the molecular mechanism of Raf activation is not completely understood (Morrison and Cutler 1997). Ras-Raf interaction is important for Raf activation, but such interaction alone is not sufficient to activate Raf in vitro. Raf activation requires additional events, such as stimulatory/inhibitory phosphorylation and association of other molecules (Morrison and Cutler 1997). Phosphorylation plays a key role in Raf activation. However, the kinase responsible for Raf activation in response to Ras or growth factors is largely unknown. Both PKC and Pak3 have been implicated to phosphorylate and activate Raf (Kolch et al. 1993; King et al. 1998). It is possible that Sur-8 may recruit a Raf-activating kinase to the complex, thereby facilitating Raf-activation. Recent genetic and biochemical data indicate that phosphorylation could also lead to Raf inactivation (Morrison and Cutler 1997; Sieburth et al. 1999; Zimmermann and Moelling 1999). Thus, it is equally possible that Sur-8 may recruit a protein phosphatase, such as PP2A (Sieburth et al. 1999), to the complex and facilitate the specific dephosphorylation of the inhibitory sites in Raf. Future biochemical studies of Sur-8 functions will provide new insights of the Ras signal transduction.

\section{Materials and methods}

Cell cultures and transfections

Human 293 cells were cultured in DMEM supplemented with 10\% (vol/ vol) FBS. Transfections of 293 cells were done by LipofectAmine (GIBCO BRL) methods in six-well plates.
Plasmid constructions

$\mathrm{H}$-Ras, K-Ras, and N-Ras cDNA were subcloned into the vector pcDNA3HA or pCDNA3. GST-tagged constructs, GST-Ras, GST-Raf, and GST-Sur-8 were obtained by subcloning into bacterial-expressing vector pGEX-KG (Guan and Dixon 1991) or mammalian-expressing vector pEBG-3X. JNK/pcDNA3HA, Supmek/pcDNA3, Raf/pRK5 Myc, pcDNA3 Flag, and Myc-tagged ERK1, Gal4-Elk-1, Gal4-LUC, and CMVlacZ were laboratory stocks. Constitutively active HA-AKT was obtained from Dr. Anne Vojtek (University of Michigan, Ann Arbor). Constitutively active HA-B-Raf/pcDNA3HA was created by site-directed mutagenesis in which Ser-364, Thr-428, and Ser-439 were replaced by alanine residues (A. Vojtek, unpubl.). Human Sur-8 cDNA was reported previously (Sieburth et al. 1998). Point mutations and deletions of Ras, Sur-8, and Raf were created by site-directed mutagenesis or PCR, and were confirmed by DNA sequencing. The plasmids used for yeast twohybrid interaction were in pVP16 or plexA-Ade vector as targets or baits (Vojtek and Hollenberg 1995), respectively.

\section{Kinase assays}

Myc-ERK1 was transfected into 293 cells. Twenty-four hours after transfections, cells were starved in serum-free medium for $12 \mathrm{hr}$. Cells were lysed in lysis buffer (10 mm Tris - $\mathrm{HCl}$ at $\mathrm{pH}$ 7.5, 2 mm EDTA, $150 \mathrm{~mm}$ $\mathrm{NaCl}, 1 \% \mathrm{NP}-40,50 \mathrm{~mm} \mathrm{NaF}, 1 \mathrm{~mm}$ sodium vanadate, $1 \mathrm{~mm}$ PMSF, 2 $\mu \mathrm{g} / \mathrm{ml}$ aprotinin, $2 \mu \mathrm{g} / \mathrm{ml}$ leupeptin). A total of $1 \mu \mathrm{g}$ of 9E10 antibody (anti-Myc) was added in each immunoprecipitation reaction. Kinase activities of ERK were measured using GST-Elk-1 as a substrate following the previously described method (Zheng and Guan 1994).

Raf-1 kinase activity was assessed by the coupled MEK/ERK activation assays according to previously described methods (Wu et al. 1996). Briefly, Flag-Raf was immunoprecipitated and used to activate $0.1 \mu \mathrm{g}$ of GST-MEK1. The activated GST-MEK1 was removed from the protein G-bound Raf and used to activate $0.1 \mu \mathrm{g}$ of GST-ERK1. Phosphorylation of GST-Elk-1 by activated GST-ERK1 was determined.

HA-JNK kinase protein was immunoprecipitated by $\alpha$-HA antibody. The procedure used to assay JNK activity was basically the same as ERK activity assay, except that GST-Jun was used as a substrate.

HA-AKT was transfected into 293 cells together with RasV12 and Sur- 8 plasmids. About 24 hr after transfection, cells were starved in serum-free DMEM overnight. Cells were then lysed in lysis buffer $(20 \mathrm{~mm}$ Tris at $\mathrm{pH} 7.5,150 \mathrm{~mm} \mathrm{NaCl}, 10 \%$ glycerol, 1\% Nonidet P-40, $10 \mathrm{~mm}$ $\mathrm{NaF}, 1 \mathrm{~mm}$ sodium Vanadate, $1 \mathrm{~mm} \mathrm{Na}_{4} \mathrm{O}_{7} \mathrm{P}_{2}, 1 \mathrm{~mm}$ PMSF, $2 \mu \mathrm{g} / \mathrm{ml}$ aprotinin, $2 \mu \mathrm{g} / \mathrm{ml}$ leupeptin). Immunoprecipitation was performed by addition of monoclonal anti-HA antibody (1-2 $\mu \mathrm{g})$. Immune complexes were collected with protein A agarose and washed three times with lysis buffer and once with kinase buffer without ATP (20 mM HEPES at pH 7.4, $10 \mathrm{~mm} \mathrm{MgCl}_{2}, 1 \mathrm{~mm}$ DTT). Kinase reaction was performed in $20 \mu \mathrm{l}$ of kinase reaction $50 \mu \mathrm{M}$ ATP (with $1.5 \mu \mathrm{m} \mathrm{Ci}\left[{ }^{32} \mathrm{P}\right] \mathrm{ATP}$ ) and $2 \mu \mathrm{g}$ of histone $2 \mathrm{~B}$ as a substrate. Reaction was incubated at $30^{\circ} \mathrm{C}$ for $20 \mathrm{~min}$ and followed by analysis on SDS-PAGE and autoradiography.

\section{Coimmunoprecipitation and Western blot}

Transfected 293 cells were lysed in IP buffer containing protease inhibitors $(10 \mathrm{~mm}$ Tris- $\mathrm{HCl}$ at $\mathrm{pH}$ 7.5, 5 mм EGTA, $150 \mathrm{~mm} \mathrm{NaCl}, 1 \%$ NP-40, $20 \mathrm{~mm} \mathrm{NaF}, 1 \mathrm{~mm}$ Sodium Vanadate, $1 \mathrm{~mm}$ PMSF, $10 \mu \mathrm{g} / \mathrm{ml}$ aprotinin, 10 $\mu \mathrm{g} / \mathrm{ml}$ leupeptin). Cell extracts were incubated with antibody for $2 \mathrm{hr}$ at $4^{\circ} \mathrm{C}$. Protein A or G beads were added in IP reactions and incubated for an additional hour. Immunoprecipitates were collected by centrifugation, washed four times with IP buffer, and analyzed by Western blot. For GST fusion proteins, cell extracts were incubated with glutathione-agarose beads directly for $1-2 \mathrm{hr}$ at $4^{\circ} \mathrm{C}$. The beads were then washed four times with lysis buffer, and eluted with $5 \mathrm{~mm}$ glutathione. The eluate was analyzed by immunoblot using indicated antibodies. For coimmunoprecipitation of endogenous proteins, exponentially growing 293 cells were used. The anti-Sur-8 antiserum was a generous gift of Dr. Mike Stern (Yale University, New Haven, CT) (Selfors et al. 1998).

For analysis of complex formation, HA-RasV12, Myc-Raf, and GSTSur- 8 were cotransfected into 293 cells. pEBG vector was cotransfected with HA-RasV12 and Myc-Raf as a control. Cell extracts were incubated with glutathione-agarose beads. GST fusion and associated proteins were eluted by $5 \mathrm{~mm}$ glutathione and subjected to immunoprecipitation with either anti-HA or anti-Myc antibodies. For experiments using His-Sur-8, nickel agarose was used in the first precipitation. The bound proteins were eluted with $50 \mathrm{~mm}$ imidazole, followed by immunoprecipitation 
Li et al.

with anti-HA antibody (for HA-Ras). Finally, the immunoprecipitated samples were analyzed by Western blot against either anti-Ras or antiRaf antibody.

In vitro binding

In vitro binding was performed in binding buffer $[50 \mathrm{~mm}$ Tris- $\mathrm{HCl}$ at $\mathrm{pH}$ 7.5, $150 \mathrm{~mm} \mathrm{NaCl}, 1.0 \mathrm{~mm}$ DTT, $1 \%$ Triton X-100, $5 \mathrm{~mm} \mathrm{MgCl} 2,25 \mu \mathrm{M}$ $\mathrm{ZnCl}_{2}$, and $0.2 \%$ (wt/vol) BSA]. Purified GST-fusion proteins or cell lysates containing GST-fusion proteins were incubated with cell lysates expressing proteins of interest. The mixture was incubated for $\sim 6 \mathrm{hr}$ and then incubated with glutathione-agarose for 1-2 $\mathrm{hr}$. The beads were washed four times with binding buffer, once with binding buffer without BSA, and then eluted with $5 \mathrm{~mm}$ glutathione and analyzed by SDS-PAGE and Western blot.

\section{Acknowledgments}

We thank Drs. Derek Sieburth and Qun Sun for the Sur-8 plasmids, Kevin Pumiglia for Raf-1 plasmids, and M. Stern for anti-soc-2 antibodies. This work was supported by grants from National Institutes of Health (K.L.G. and M.H.) and the MacArthur Fellowship (K.L.G.).

The publication costs of this article were defrayed in part by payment of page charges. This article must therefore be hereby marked "advertisement" in accordance with 18 USC section 1734 solely to indicate this fact.

\section{References}

Chang, H.C., F.D. Karim, E.M. O'Neill, I. Rebay, N.M. Solomon, M Therrien, D.A. Wassarman, T. Wolff, and G.M. Rubin. 1994. Ras signal transduction pathway in Drosophila eye development. Cold Spring Harb. Symp. Quant. Biol. 59: 147-153.

Derijard, B., M. Hibi, I.H. Wu, T. Barrett, B. Su, T. Deng, M. Karin, and R.J. Davis. 1994. JNK1: A protein kinase stimulated by UV light and Ha-Ras that binds and phosphorylates the c-Jun activation domain. Cell 76: 1025-1037.

Field, J., H.P. Xu, T. Michaeli, R. Ballester, P. Sass, M. Wigler, and J. Colicelli. 1990. Mutations of the adenylyl cyclase gene that block RAS function in Saccharomyces cerevisiae. Science 247: 464-467.

Guan, K.L. and J.E. Dixon. 1991. Eukaryotic proteins expressed in Escherichia coli: An improved thrombin cleavage and purification procedure of fusion proteins with glutathione S-transferase. Anal. Biochem. 192: 262-267.

Howe, L.R., S.J. Leevers, N. Gomez, S. Nakielny, P. Cohen, and C.J. Marshall. 1992. Activation of the MAP kinase pathway by the protein kinase raf. Cell 71: 335-342.

Kataoka, T., D. Broek, and M. Wigler. 1985. DNA sequence and characterization of the $\mathrm{S}$. cerevisiae gene encoding adenylate cyclase. Cell 43: 493-505

Katz, M.E. and F. McCormick. 1997. Signal transduction from multiple Ras effectors. Curr. Opin. Genet. Dev. 7: 75-79.

King, A.J., H. Sun, B. Diaz, D. Barnard, W. Miao, S. Bagrodia, and M.S Marshall. 1998. The protein kinase Pak3 positively regulates Raf-1 activity through phosphorylation of serine 338. Nature 396: 180-183.

Kobe, B. and J. Deisenhofer. 1993. Crystal structure of porcine ribonuclease inhibitor, a protein with leucine-rich repeats. Nature 366: 751756.

Kolch, W., G. Heidecker, G. Kochs, R. Hummel, H. Vahidi, H. Mischak, G. Finkenzeller, D. Marme, and U.R. Rapp. 1993. Protein kinase C alpha activates RAF-1 by direct phosphorylation. Nature 364: 249252.

Kornfeld, K., D.B. Hom, and H.R. Horvitz. 1995. The ksr-1 gene encodes a novel protein kinase involved in Ras-mediated signaling in C. elegans. Cell 83: 903-913.

Kyriakis, J.M., H. App, X.F. Zhang, P. Banerjee, D.L. Brautigan, U.R. Rapp, and J. Avruch. 1992. Raf-1 activates MAP kinase-kinase. Nature 358: 417-421.

Kyriakis, J.M., P. Banerjee, E. Nikolakaki, T. Dai, E.A. Rubie, M.F. Ahmad, J. Avruch, and J.R. Woodgett. 1994. The stress-activated protein kinase subfamily of c-Jun kinases. Nature 369: 156-160.

Macara, I.G., K.M. Lounsbury, S.A. Richards, C. McKiernan, and D. BarSagi. 1996. The Ras superfamily of GTPases. FASEB J. 10: 625-630.
Mansour, S.J., W.T. Matten, A.S. Hermann, J.M. Candia, S. Rong, K. Fukasawa, G.F. Vande Woude, and N.G. Ahn. 1994. Transformation of mammalian cells by constitutively active MAP kinase kinase. Science 265: 966-970.

Marte, B.M. and J. Downward. 1997. PKB/Akt: Connecting phosphoinositide 3-kinase to cell survival and beyond. Trends Biochem. Sci. 22: $355-358$.

Morrison, D.K. and R.E. Cutler. 1997. The complexity of Raf-1 regulation. Curr. Opin. Cell. Biol. 9: 174-179.

Robinson, M.J. and M.H. Cobb. 1997. Mitogen-activated protein kinase pathways. Curr. Opin. Cell. Biol. 9: 180-186.

Rodriguez-Viciana, P., P.H. Warne, R. Dhand, B. Vanhaesebroeck, I. Gout, M.J. Fry, M.D. Waterfield, and J. Downward. 1994. Phosphatidylinositol-3-OH kinase as a direct target of Ras. Nature 370: 527 532.

Selfors, L.M., J.L. Schutzman, C.Z. Borland, and M.J. Stern. 1998. soc-2 encodes a leucine-rich repeat protein implicated in fibroblast growth factor receptor signaling. Proc. Natl. Acad. Sci. 95: 6903-6908.

Sieburth, D.S., Q. Sun, and M. Han. 1998. SUR-8, a conserved Ras-binding protein with leucine-rich repeats, positively regulates Ras-mediated signaling in C. elegans. Cell 94: 119-130.

Sieburth, D.S., M. Sundaram, R.M. Howard, and M. Han. 1999. A PP2A regulatory subunit positively regulates Ras-mediated signaling during Caenorhabditis elegans vulval induction. Genes \& Dev. 13: 2562-2569.

Sternberg, P.W. and M. Han. 1998. Genetics of RAS signaling in C. elegans. Trends Genet. 14: 466-472.

Sundaram, M. and M. Han. 1995. The C. elegans ksr-1 gene encodes a novel Raf-related kinase involved in Ras-mediated signal transduction. Cell 83: 889-901.

Suzuki, N., H.R. Choe, Y. Nishida, Y. Yamawaki-Kataoka, S. Ohnishi, T Tamaoki, and T. Kataoka. 1990. Leucine-rich repeats and carboxyl terminus are required for interaction of yeast adenylate cyclase with RAS proteins. Proc. Nat1. Acad. Sci. 87: 8711-8715.

Urano, T., R. Emkey, and L.A. Feig. 1996. Ral-GTPases mediate a distinct downstream signaling pathway from Ras that facilitates cellular transformation. EMBO J. 15: 810-816.

van der Geer, P., T. Hunter, and R.A. Lindberg. 1994. Receptor proteintyrosine kinases and their signal transduction pathways. Annu. Rev. Cell. Biol. 10: 251-337.

Vojtek, A.B. and C.J. Der. 1998. Increasing complexity of the Ras signaling pathway. J. Biol. Chem. 273: 19925-19928.

Vojtek, A.B. and S.M. Hollenberg. 1995. Ras-Raf interaction: Two-hybrid analysis. Methods Enzymol. 255: 331-342.

Voitek, A.B., S.M. Hollenberg, and J.A. Cooper. 1993. Mammalian Ras interacts directly with the serine/threonine kinase Raf. Cell 74: 205 214.

Wu, X., S.J. Noh, G. Zhou, J.E. Dixon, and K.L. Guan. 1996. Selective activation of MEK1 but not MEK2 by A-Raf from epidermal growth factor-stimulated Hela cells. I. Biol. Chem. 271: 3265-3271.

Zheng, C.F. and K.L. Guan. 1994. Activation of MEK family kinases requires phosphorylation of two conserved Ser/Thr residues. EMBO J. 13: $1123-1131$.

Zimmermann, S. and K. Moelling. 1999. Phosphorylation and regulation of Raf by Akt (protein kinase B). Science 286: 1741-1744. 


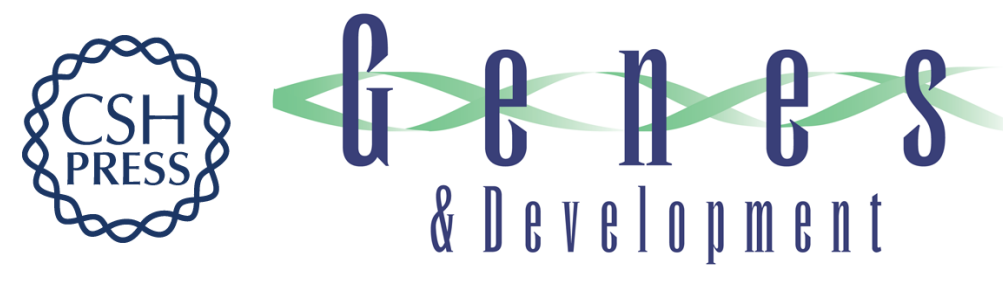

\section{The leucine-rich repeat protein SUR-8 enhances MAP kinase activation and forms a complex with Ras and Raf}

Weiquan Li, Min Han and Kun-Liang Guan

Genes Dev. 2000, 14:

Access the most recent version at doi:10.1101/gad.14.8.895

References This article cites 33 articles, 9 of which can be accessed free at: http://genesdev.cshlp.org/content/14/8/895.full.html\#ref-list-1

License

Email Alerting

Receive free email alerts when new articles cite this article - sign up in the box at the top Service right corner of the article or click here.

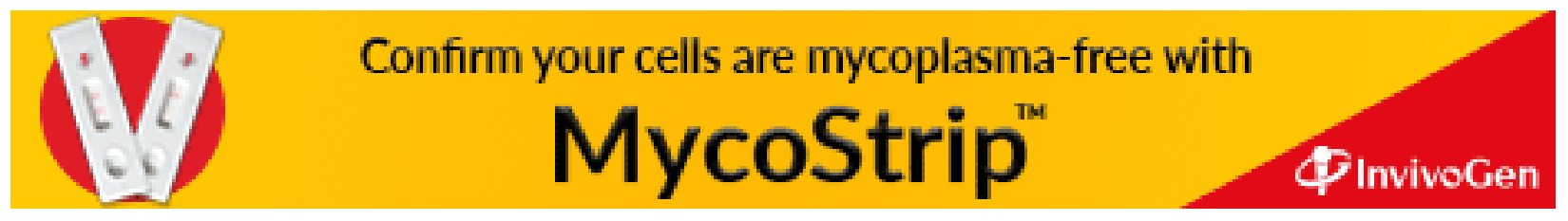

